\title{
Structural homogeneity in microbial lipases.
}

\author{
Swati Verma ${ }^{1}$, Gautam Kumar Meghwanshi ${ }^{1}$, Rajender Kumar ${ }^{2 *}$ \\ ${ }^{1}$ Department of Microbiology, Maharaja Ganga Singh University, Bikaner-334004, India \\ ${ }^{2}$ Present address: Department of Clinical Microbiology, Umea University, SE-90185 Umea, Sweden
}

Accepted on February 2, 2018

\section{Editorial}

Enzyme lipase (triacylglycerol hydrolases E.C.3.1.1.3) catalyzes the hydrolysis of triacylglycerols to glycerol and free fatty acids. In recent years, progressively more consideration is being rewarded to lipases produced by microorganisms. In nature, some of the most important lipase-producing bacteria are Pseudomonas, Bacillus, Staphylococcus, etc., and fungi are Rhizopus sp., Aspergillus, Penicillium, Geotrichum, Mucor, Rhizomucor, etc. [1,2]. The bacterial lipases from Bacillus sp. exhibit interesting properties that make them potential candidates for biotechnological applications [1-3]. Bacillus alcalophilus are the most common lipase producing strains. Lipases are classified into different families based on the amino-acid sequences similarity and their biological properties [4]. Microbial lipases are relatively stable and are capable of catalyzing a variety of reactions; they hold immense potential for diverse industrial applications.

Primary protein sequences of lipases are available from a number of different microorganisms, including, archaea, bacteria and fungi, and show considerable sequence diversity, ranging from as little as $5 \%$ to as much as $90 \%$. Total 85 unique presentative structures (Bacteria: 43; Eukaryota: 40; and Archaea: 2) of true lipases (triacylglycerol hydrolases) are reported in Protein Data Bank (PDB, http://www.rcsb.org) accessed in December 2017. At 30\% sequences identity, only 22 unique presentative structures (Bacteria: 12; Eukaryota: 9; and Archaea: 1) of lipase have been found. In recent years, structural information and computational molecular modeling studies demonstrated their mechanistic properties $[5,6]$. The $3 \mathrm{D}$ structure of lipases usually have a common $\alpha / \beta$ hydrolase fold topology with mostly parallel $\beta$-sheets, flanked on both sides by $\alpha$-helices [7]. A highly conserved catalytic triad (Serine, Aspartic/Glutamic acid and Histidine) houses the active site of lipase. The nucleophilic active site serine residue is always found to be in a short loop between a strand and a $\alpha$-helix. This residue is usually found in the conserved pentapeptide sequence Gly-x-Ser-x-Gly, located in the hydrophobic pocket of the active site making it suitable for accepting triglycerides and other hydrophobic substrates. The active site of many lipases is covered by one or more short $\alpha$-helixes, which form a lid/flap. The lid moves away upon interaction with the substrate and that conformational change results in the activation of these enzymes at an oil-water interface.

Discovery of novel extremophilic microbes and their enzymes has an important impact on biocatalysis and in the field industrial biotechnology. Thermal stability is one of the most desirable characteristics in the search for novel lipases. Some important requisites for thermostability of enzymes are a relatively small hydrophobic surface, exposed $\mathrm{N}$ - and $\mathrm{C}$ termini, loops that are fixated near the center of the enzyme, strong ion-pairing, e. g. with arginine residues, hydrogen and disulfide bonds, interactions between aromatic pairs and hydrophobic interactions [8]. Normally, an increase in internal hydrophobicity and stabilization of the secondary structure together with compactness and rigidity of the overall structure enhance protein thermostability $[9,10]$. There are a number of thermophilic lipases with temperature optima of $70^{\circ} \mathrm{C}$ and more; however, they are not stable for long time at these temperatures.
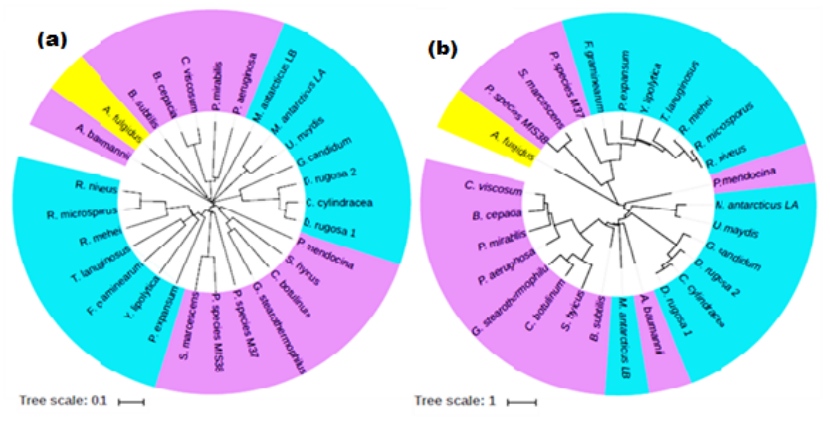

Figure 1. An overview of diversity of microbial lipase (a) at sequence level analysis (b) at structural level, structural distance calculated by sequence-independent alignment of the local backbone similarity matching method of the mammoth algorithm by max cluster program.

The comprehensive comparative structural analysis of microbial lipases has been performed to explore their conservations and diversity that reflect their stabilities and enzymatic activities. The thorough structural elucidation of microbial lipases has been carried out from the reported structures and their amino acid sequences of different microbial lipases, isolated from bacteria, archea and fungi. More recently resolved crystal structures of thermostable lipases, one from marine Streptomyces (PDB ID: 5H6B) and another an extracellular lipase from Streptomyces rimosus (PDB ID: $5 \mathrm{MAL}$ ) were not include (published after analysis). The results based on sequence and sequence-independent structural alignments were further used to construct a guide tree using neighbour-joining algorithm. The comparative results for amino acid sequence and structural features of lipases showed that although they have diversity at the level of sequences, but structurally they are highly conserved and adopted a specific structural topology. Those structural similarities are clearly shown in separated groups (Figure 1). In these unique homologs, structural topology maintains enzymatic activities especially for extremophilic microbial lipases. The structural key points reflect the activity of liapses at different environment conditions. Structurally different architectures 
might play an important role in their thermal stabilities and enzymatic activities [11-14]. Highly conserved pentapeptide sequence Ala/Gly-x-Ser-x-Gly and some other conserved regions reported in extremophilic lipases. This information would enrich the current knowledge on lipases with respect to their structure and function relationship and industrial applications for which they may be used. Further, the deduced information on structure-function relationship may be used to amend the lipase activity and stability as per the requirements of the particular industrial processes.

\section{References}

1. Gupta R, Gupta N, Rathi P. Bacterial lipases: an overview of production, purification and biochemical properties. Appl Microbiol Biotechnol. 2004;64:763-81.

2. Treichel H, de Oliveira D, Mazutti MA, et al. A review on microbial lipases production. Food and Bioprocess Technol. 2010;3:182-96.

3. Kirk O, Borchert TV, Fuglsang CC. Industrial enzyme applications. Curr Opin Biotechnol. 2002;13:345-51.

4. Arpigny JL, Jaeger KE. Bacterial lipolytic enzymes: classification and properties. Biochem J. 1999;343:177-83.

5. Kaschner M, Schillinger O, Fettweiss T, et al. combination of mutational and computational scanning guides the design of an artificial ligand-binding controlled lipase. Sci Rep. 2017;7:42592.

6. Barbe S, Cortés J, Simeon T, et al. A mixed molecular modeling-robotics approach to investigate lipase large molecular motions. Proteins. 2011;79:2517-29.

7. Ollis DL, Shea E, Cygler M, et al. The $\alpha / \beta$ hydrolase fold. Protein Eng. 1992; 5:197-211.
8. Jaenicke R, Bohm G. The stability of proteins in extreme environments. Curr Opin Struct Biol. 1998;8:738-48.

9. Jaenicke R. How do proteins acquire their three dimensional structure and stability? Natur wissen schaften. 1996:83:544-54.

10. Vieille C, Zeikus G. Hyperthermopilic enzymes: Source, uses, and molecular mechanism for thermostability. Microbiol Mol Biol Rev. 2001;65:1-43.

11. Sharma PK, Kumar R, Kumar R, et al. Engineering of a metagenome derived lipase towards thermal tolerance: effect of aspargine to lysine mutation on the protein surface. 2011;491:264-71.

12. Adams MWW, Kelly RM. Enzymes from microorganisms in extreme environments. Chem and Eng News. 1995;73:32-42.

13. Demirjian D, Morís-Varas F, Cassidy C. Enzymes from extremophiles. Curr Opin Chem Biol. 2001;5:144-51.

14. Spiller B. Gershenson A, Stevens FH, et al. A structural view of evolutionary divergence. Proc Natl Acad Sci. 1999;96:12305-10.

\section{*Correspondence to:}

Rajender Kumar

Present address: Department of Clinical Microbiology

Umea University, Sweden

E-mail: rajbioinformaticshau@gmail.com 